\title{
Características de desempenho e eficiência alimentar de touros Purunã em crescimento de três classes de consumo alimentar residual
}

\author{
[Performance and feed efficiency traits of Purunã growing bulls of three \\ groups of residual feed intake] \\ S.R. Fernandes ${ }^{1,5}$, I.C.B. Stieven ${ }^{1}$, G.F. Zanetti ${ }^{1}$, L.H. Kowalski ${ }^{2,5}$, P. Rossi Junior ${ }^{3}$, \\ J.L. Moletta ${ }^{4}$, R. Almeida ${ }^{3}$ \\ ${ }^{1}$ Pós-graduação - Universidade Federal do Paraná - UFPR - Curitiba, PR \\ ${ }^{2}$ Pós-graduação - Universidade Federal do Paraná - Palotina, PR \\ ${ }^{3}$ Universidade Federal do Paraná - UFPR - Curitiba, PR \\ ${ }^{4}$ Instituto Agronômico do Paraná - IAPAR - Estação Experimental Fazenda Modelo - Ponta Grossa, PR \\ ${ }^{5}$ Bolsista CAPES
}

\begin{abstract}
RESUMO
Objetivou-se avaliar as características de desempenho, consumo e eficiência alimentar de touros Purunã em crescimento de três classes de consumo alimentar residual (CAR): eficiente, intermediária e ineficiente. Foram avaliados 110 tourinhos com média de 10 meses de idade e $233 \mathrm{~kg}$ de peso corporal (PC), distribuídos aleatoriamente em baias individuais em confinamento. A dieta, em base de matéria seca (MS), foi composta por $420 \mathrm{~g} / \mathrm{kg}$ de concentrado e $580 \mathrm{~g} / \mathrm{kg}$ de silagem de milho, e fornecida à vontade. O consumo de MS diário e o ganho médio diário (GMD) foram avaliados durante 112 dias. O PC inicial e final e o GMD foram semelhantes entre as classes de eficiência e apresentaram valores médios de $233 \mathrm{~kg}$, $360 \mathrm{~kg}$ e $1,14 \mathrm{~kg} /$ dia. Valores médios de 7,$19 ; 7,62$ e $8,10 \mathrm{~kg} /$ dia para consumo de MS e $-048 ;-0,01$ e 0,48kg MS/dia para CAR foram observados nas classes eficiente, intermediária e ineficiente. A conversão alimentar (CA) e a eficiência alimentar bruta (EAB) foram semelhantes entre as classes eficiente e intermediária, e apresentaram média de $6,65 \mathrm{~kg} \mathrm{MS} / \mathrm{kg}$ ganho e $153 \mathrm{~g}$ ganho/ $\mathrm{kg} \mathrm{MS}$. A classe ineficiente apresentou maior CA e menor EAB, com valores de $7,40 \mathrm{~kg} \mathrm{MS} / \mathrm{kg}$ ganho e $138 \mathrm{~g}$ ganho/kg MS. A eficiência parcial de crescimento (EPC) diferiu entre as classes de eficiência e apresentou valores médios de 294; 277 e 255g ganho/kg MS para crescimento nas classes eficiente, intermediária e ineficiente. O CAR apresentou correlação moderada a alta com CA, EAB e EPC, e a diminuição do CAR levou à redução da CA em 12,2\%, e aumento da EAB e EPC em 13,3\% e 15,5\%. Tourinhos Purunã eficientes para CAR consomem menos alimento, mas mantêm o mesmo nível de produção que os ineficientes. Assim, a classificação com base no CAR permite a identificação de animais com potencial para melhorar a eficiência produtiva do rebanho.
\end{abstract}

Palavras-chave: consumo de matéria seca, conversão alimentar, eficiência parcial de crescimento, ganho médio diário

\begin{abstract}
This trial aimed to evaluate the performance, feed intake and feed efficiency traits of Purunã growing bulls of three groups of residual feed intake (RFI): efficient, intermediary and inefficient. There were used 110 growing bulls with average of 10 months of age and $233 \mathrm{~kg}$ of body weight (BW) which were randomly allocated in individual feedlot pens. The diet was composed, in dry matter (DM) basis, by $420 \mathrm{~g} / \mathrm{kg}$ of concentrate and $580 \mathrm{~g} / \mathrm{kg}$ of corn silage, and was offered ad libitum. Daily dry matter intake (DMI) and average daily gain $(A D G)$ were assessed during 112 days. Initial and final BW and ADG not differed between the efficiency groups and showed mean values of $233 \mathrm{~kg}, 360 \mathrm{~kg}$ and $1.14 \mathrm{~kg} / \mathrm{d}$. Mean values of $7.19,7.62$ and $8.10 \mathrm{~kg} / \mathrm{d}$ to DMI and $-0.48,-0.01$ and $0.48 \mathrm{~kg}$ DM/d to RFI were observed in
\end{abstract}

Recebido em 1 de outubro de 2012

Aceito em 30 de julho de 2013

E-mail: srfernandes83@gmail.com 
efficient, intermediary and inefficient groups. Feed conversion ratio (FCR) and gross feed efficiency (GFE) not differed between efficient and intermediary groups, which showed means of $6.65 \mathrm{~kg} D M / \mathrm{kg}$ gain and $153 \mathrm{~g}$ gain $/ \mathrm{kg}$ DM. Inefficient group showed higher FCR and lower GFE, with mean values of $7.40 \mathrm{~kg} \mathrm{DM} / \mathrm{kg}$ gain and $138 \mathrm{~g}$ gain $/ \mathrm{kg}$ DM. Partial efficiency of growth (PEG) differed between the efficiency groups and showed means of 294, 277 and $255 \mathrm{~g}$ gain $/ \mathrm{kg}$ DM for growth in efficient, intermediary and inefficient groups. RFI showed moderate to high correlations with FCR, GFE and PEG, and decrease of RFI resulted in decrease of FCR in 12.2\%, increase of GFE in $13.3 \%$ and increase of PEG in 15.5\%. Efficient Puruna growing bulls (low RFI) present lower feed intake, but maintains the same production level that inefficient ones (high RFI). Thus, RFI classification allows identifying animals with potential to improve the productive efficiency of herd.

Keywords: average daily gain, dry matter intake, feed conversion ratio, partial efficiency of growth

\section{INTRODUÇÃO}

O consumo alimentar residual (CAR) é uma característica mensurada em avaliações de eficiência alimentar de bovinos de corte. Essa característica é definida como a diferença entre o consumo de alimento observado e o estimado em função do peso metabólico (PM) e do ganho médio diário (GMD), mensurados em intervalo de tempo pré-determinado (Koch et al., 1963). Portanto, os animais mais eficientes consomem menos alimento que o estimado, apresentando valores negativos de CAR.

O CAR é fenotipicamente independente do PM e do GMD, que são utilizados para estimar o consumo de alimento. A variação dessa característica entre indivíduos de um mesmo grupo está associada, principalmente, aos gastos energéticos com processos fisiológicos (Castro Bulle et al., 2007). Com a diminuição da necessidade de ingestão de energia em animais eficientes (CAR negativo), é possível manter o desempenho produtivo do rebanho com menor quantidade de alimento, o que pode reduzir os custos com alimentação, aumentar a produtividade e, ainda, melhorar as condições ambientais pela redução da emissão de metano (Hegarty et al., 2007).

No Brasil, a característica CAR tem sido mensurada e utilizada na avaliação da eficiência alimentar de bovinos Nelore. Essa avaliação tem sido realizada em tourinhos recém-desmamados (Lucila Sobrinho et al., 2011) e em touros adultos submetidos a provas de desempenho (Santana et al., 2012). Nesses estudos não foram observadas diferenças no peso corporal (PC) médio e no GMD entre animais eficientes (CAR negativo) e ineficientes (CAR positivo), mas os primeiros apresentaram menor consumo de alimento e, por consequência, maior eficiência produtiva.

Os rebanhos de corte na região Sul são formados, principalmente, por animais de raças taurinas. Nessa condição, o Instituto Agronômico do Paraná (IAPAR), utilizando raças com potencial para produção de carne existentes nesse Estado, desenvolveu o bovino composto Purunã. Desenvolvida e selecionada há 30 anos no IAPAR, essa raça é formada por $1 / 4$ Angus, 1/4 Caracu, 1/4 Charolês e 1/4 Canchim. Esses animais apresentam alto potencial de ganho de peso, precocidade na deposição de gordura de cobertura e alto rendimento de carcaça (Kuss et al., 2008). Diante da escassez de informações sobre a raça Purunã, estudos de desempenho e eficiência alimentar são necessários e podem contribuir para a melhoria dos índices zootécnicos e econômicos nos sistemas de produção que utilizam essa raça.

Objetivou-se, com o presente estudo, avaliar as características de desempenho, consumo e eficiência alimentar de touros Purunã em crescimento, classificados como eficientes, intermediários e ineficientes para CAR.

\section{MATERIAL E MÉTODOS}

Dois experimentos foram realizados em 2011 e 2012 na Estação Experimental Fazenda Modelo (EEFM) do IAPAR, localizada em Ponta Grossa, PR. Nos dois anos de avaliação, o período experimental se estendeu de abril a agosto.

Foram avaliados 110 tourinhos da raça Purunã, filhos de 66 touros e 105 vacas utilizadas em cruzamentos para a obtenção de animais puros da raça Purunã, todos provenientes da EEFM do 
IAPAR. Em 2011 e 2012 foram avaliados 60 e 50 animais, respectivamente.

O manejo pré-experimental foi padronizado nos dois anos de avaliação, em que os tourinhos foram desmamados com $8 \pm 1,1$ meses de idade e $201 \pm 35,3 \mathrm{~kg}$ de PC. Após o desmame, os animais permaneceram em pastagem de hemártria (Hemarthria altissima cv. Florida) com suplementação concentrada até o início do período de adaptação ao confinamento experimental. $\mathrm{O}$ suplemento concentrado foi ofertado a $0,6 \%$ PC em matéria seca (MS)/animal/dia.
O período de adaptação teve início 60 dias após o desmame, quando os tourinhos apresentavam $10 \pm 1,1$ meses de idade. Os animais foram distribuídos aleatoriamente em baias individuais em confinamento coberto, e o período de adaptação às instalações e à dieta experimental foi de 14 dias.

A dieta, em base de MS, foi composta por $420 \mathrm{~g} / \mathrm{kg}$ de concentrado e $580 \mathrm{~g} / \mathrm{kg}$ de silagem de milho (Tab. 1). O concentrado, em base de matéria natural (MN), foi composto por $150 \mathrm{~g} / \mathrm{kg}$ de farelo de soja, $180 \mathrm{~g} / \mathrm{kg}$ de soja grão, $650 \mathrm{~g} / \mathrm{kg}$ de milho grão moído e $20 \mathrm{~g} / \mathrm{kg}$ de suplemento mineral (Allfós 60 ${ }^{\circledR}$ em 2011 e Fosbovi $30^{\circledR}$ em 2012).

Tabela 1. Teores médios de matéria seca ( $\mathrm{g} / \mathrm{kg} \mathrm{MN})$ e composição nutricional (g/kg MS) do concentrado, da silagem de milho e da dieta total ofertada aos animais nos dois anos de avaliação

\begin{tabular}{|c|c|c|c|c|c|c|}
\hline \multirow[b]{2}{*}{ Composição $^{1}$} & \multicolumn{3}{|c|}{2011} & \multicolumn{3}{|c|}{2012} \\
\hline & Concentrado & $\begin{array}{l}\text { Silagem } \\
\text { de Milho }\end{array}$ & $\begin{array}{l}\text { Dieta } \\
\text { Total }\end{array}$ & Concentrado & $\begin{array}{l}\text { Silagem } \\
\text { de Milho }\end{array}$ & $\begin{array}{l}\text { Dieta } \\
\text { Total }\end{array}$ \\
\hline $\mathrm{MS}(\mathrm{g} / \mathrm{kg} \mathrm{MN})$ & 880,2 & 286,2 & 400,5 & 888,9 & 281,6 & 398,7 \\
\hline PB (g/kg MS) & 193,4 & 73,1 & 124,0 & 203,5 & 83,4 & 135,0 \\
\hline $\mathrm{EE}(\mathrm{g} / \mathrm{kg} \mathrm{MS})$ & 81,8 & 21,0 & 46,7 & 73,9 & 33,0 & 50,6 \\
\hline FDN (g/kg MS) & 94,2 & 562,0 & 364,1 & 94,6 & 534,6 & 345,4 \\
\hline FDA (g/kg MS) & 79,2 & 335,2 & 226,9 & 35,1 & 297,1 & 184,4 \\
\hline LIG (g/kg MS) & 9,1 & 27,1 & 19,5 & 2,5 & 38,6 & 23,1 \\
\hline RM (g/kg MS) & 45,6 & 30,2 & 36,7 & 44,4 & 41,7 & 42,9 \\
\hline $\mathrm{Ca}(\mathrm{g} / \mathrm{kg} \mathrm{MS})$ & 5,1 & 2,4 & 3,5 & 4,3 & 3,2 & 3,7 \\
\hline $\mathrm{P}(\mathrm{g} / \mathrm{kg} \mathrm{MS})$ & 5,1 & 2,1 & 3,4 & 5,8 & 2,1 & 3,7 \\
\hline EM (Mcal/kg MS) & 3,35 & 2,37 & 2,78 & 3,37 & 2,35 & 2,79 \\
\hline
\end{tabular}

${ }^{1}$ MS: matéria seca; MN: matéria natural; PB: proteína bruta; EE: extrato etéreo; FDN: fibra em detergente neutro; FDA: fibra em detergente ácido; LIG: lignina; RM: resíduo mineral; Ca: cálcio; P: fósforo; EM: energia metabolizável. PB, EE, LIG, RM, Ca e P foram determinados conforme Silva e Queiroz (2002); FDN e FDA foram determinados de acordo com Van Soest et al. (1991); EM calculada com base nas equações propostas no NRC (2001).

Os animais foram alimentados à vontade e receberam a dieta na forma de ração total misturada (RTM), fracionada em duas ofertas diárias (10 e 15h). O ajuste da quantidade de alimento fornecido foi realizado diariamente para cada animal, mantendo-se as sobras entre $10 \mathrm{e}$ $15 \%$ da quantidade ofertada.

O período experimental teve início com a mensuração do consumo alimentar e do desempenho individual dos animais, que compreendeu 112 dias. Os animais iniciaram os experimentos devidamente vacinados, e foram vermifugados (Ivermectina a $3,15 \%-1 \mathrm{~mL} / 50 \mathrm{~kg}$ PC) nos dias experimentais 0 e 84 .
$\mathrm{O}$ consumo diário de matéria seca $\left(\mathrm{CMS}_{\mathrm{OBS}}\right)$ foi calculado pela diferença entre a quantidade de alimento fornecido e a quantidade de sobras. Estas foram colhidas, pesadas e amostradas (alíquotas que representavam cerca de $10 \%$ do peso) diariamente, formando-se uma amostra composta das sobras de cada animal por semana. As amostras compostas permaneceram congeladas no período de amostragem. Ao final de cada semana, essas amostras foram descongeladas, homogeneizadas e uma subamostra (cerca de $350 \mathrm{~g}$ ) foi retirada e seca em estufa de ventilação forçada a $65^{\circ} \mathrm{C}$ até atingir peso constante. Após a secagem, as amostras foram pesadas e o teor de MS foi calculado. Utilizou-se o teor médio de MS das sobras para 
corrigir o consumo MS de cada animal no período experimental.

As pesagens foram realizadas a cada 28 dias, após jejum alimentar de 16 horas. O GMD foi calculado por regressão linear do PC em função do dia experimental nas datas de pesagem $(0,28$, 56, 84 e 112). O modelo da equação de regressão correspondeu a $Y_{i}=\beta_{0}+\beta_{1} X$, onde $Y_{i}=$ peso corporal $(\mathrm{kg})$ no dia experimental i; $\beta_{0}=$ intercepto da regressão; $\beta_{1}=$ ganho médio diário $(\mathrm{kg} / \mathrm{dia}) ; \mathrm{X}=$ dia experimental. $\mathrm{O}$ coeficiente de determinação médio $\left(\mathrm{R}^{2}\right)$ das equações de regressão correspondeu a 0,98 , indicando que os tourinhos apresentaram padrão de crescimento normal nos dois anos de avaliação.

A partir do $\mathrm{CMS}_{\mathrm{OBS}}$, GMD e do $\mathrm{PC}$ médio individual dos animais nos dois anos de avaliação, foram calculados: $\mathrm{CMS}_{\mathrm{PC}}$ - obtido pela razão entre $\mathrm{CMS}_{\mathrm{OBS}}(\mathrm{kg} / \mathrm{dia})$ e o $\mathrm{PC}$ médio (kg), expresso em \% PC/dia; $\mathrm{CMS}_{\mathrm{PMM}}$ - obtido pela razão entre $\mathrm{CMS}_{\mathrm{OBS}}(\mathrm{kg} / \mathrm{dia})$ e o peso médio metabólico $\left(\mathrm{PMM}=\mathrm{PC}^{0,75}\right)$, expresso em $\%$ $\mathrm{PMM} /$ dia; $\mathrm{CMS}_{\mathrm{MANT}}$ - consumo de MS estimado para mantença $(\mathrm{kg} / \mathrm{dia})$, corresponde à razão entre a necessidade de energia diária para mantença $\left(\mathrm{E}_{\mathrm{M}}, \mathrm{Mcal} / \mathrm{dia}\right)$, calculada conforme proposto por Lofgreen e Garrett (1968), e o teor de energia líquida da dieta para mantença $\left(\mathrm{EL}_{\mathrm{M}}\right.$, Mcal/kg MS), estimada de acordo com Zinn e Shen (1998); $\mathrm{CMS}_{\mathrm{GANHO}}$ - consumo de MS estimado para crescimento ( $\mathrm{kg} / \mathrm{dia})$, é a diferença entre $\mathrm{CMS}_{\mathrm{OBS}}$ e $\mathrm{CMS}_{\mathrm{MANT}}$; e $\mathrm{CMS}_{\mathrm{EST}}$ - consumo de MS estimado (kg/dia), é calculado pela equação obtida a partir da regressão do $\mathrm{CMS}_{\mathrm{OBS}}$ (kg/dia) em função do GMD (kg/dia) e do PMM $\left(\mathrm{kg}^{0,75}\right)$, conforme proposto por Koch et al. (1963). O modelo da equação de predição de consumo de $\mathrm{MS}$ foi $\mathrm{Y}_{\mathrm{i}}=\beta_{0}+\beta_{1} \mathrm{PMM}_{\mathrm{i}}+$ $\beta_{2} \mathrm{GMD}_{\mathrm{i}}$, onde $\mathrm{Y}_{\mathrm{i}}=\mathrm{CMS}_{\mathrm{EST}}(\mathrm{kg} / \mathrm{dia})$ do animal i; $\beta_{0}=$ intercepto da regressão; $\beta_{1}=$ coeficiente parcial da regressão para $\mathrm{PMM} ; \mathrm{PMM}_{\mathrm{i}}=\mathrm{PMM}$ $\left(\mathrm{kg}^{0,75}\right)$ do animal $\mathrm{i} ; \beta_{2}$ coeficiente parcial da regressão para GMD; $\mathrm{GMD}_{\mathrm{i}}=\mathrm{GMD}(\mathrm{kg} / \mathrm{dia})$ do animal i.

As medidas de consumo alimentar e de desempenho foram utilizadas para calcular as seguintes medidas de eficiência alimentar: CA conversão alimentar, razão entre $\mathrm{CMS}_{\mathrm{OBS}}$ (kg/dia) e GMD (kg/dia), expressa em $\mathrm{kg} \mathrm{MS} / \mathrm{kg}$ ganho; EAB - eficiência alimentar bruta, inverso da CA e calculada pela razão entre GMD (kg/dia) e $\mathrm{CMS}_{\mathrm{OBS}}(\mathrm{kg} / \mathrm{dia})$, expressa em $\mathrm{g}$ ganho/kg MS; EMant - eficiência de mantença, obtida pela razão entre GMD (kg/dia) e $\mathrm{CMS}_{\text {MANT }}(\mathrm{kg} / \mathrm{dia})$, expressa em g ganho/kg MS para mantença; EPC - eficiência parcial de crescimento, razão entre GMD (kg/dia) e $\mathrm{CMS}_{\mathrm{GANHO}}(\mathrm{kg} / \mathrm{dia})$, expressa em g ganho/kg MS para crescimento; TCR - taxa de crescimento relativo, calculada pela fórmula $\mathrm{TCR}=$ $100 *(\log \mathrm{PF}-\log \mathrm{PI}) / \mathrm{PE}$, onde $\mathrm{PF}=$ peso corporal final $(\mathrm{kg}), \mathrm{PI}=$ peso corporal inicial $(\mathrm{kg})$ e $\mathrm{PE}=$ período experimental (dias), expressa em \%/dia (Fitzhugh Junior e Taylor, 1971); TK taxa de Kleiber, calculada pela fórmula $\mathrm{TK}=$ GMD/PMM, expressa em $\mathrm{kg}$ ganho/kg ${ }^{0,75}$ (Kleiber, 1947); e CAR - consumo alimentar residual, calculada pela fórmula $\mathrm{CAR}=\mathrm{CMS}_{\mathrm{OBS}}$ - $\mathrm{CMS}_{\mathrm{EST}}$, expressa em kg MS/dia (Koch et al., 1963).

A equação de predição de consumo de MS $\left(\mathrm{CMS}_{\mathrm{EST}}\right)$ foi gerada pelo PROC GLM no programa SAS (Statistical Analysis System, versão 9.0), sendo considerado o efeito fixo de ano. $\mathrm{O} \mathrm{CMS}_{\mathrm{EST}}$ foi calculado para cada animal pela equação $\mathrm{CMS}_{\mathrm{EST}}=-1,105+\mathrm{FAI}_{\mathrm{ANO}}+$ $0,091 * \mathrm{PMM}+1,853 * \mathrm{GMD}\left(\mathrm{R}^{2}=0,87\right)$, onde $\mathrm{FAI}_{\mathrm{ANO}}$ corresponde ao fator de ajuste do intercepto para ano de avaliação, sendo $\mathrm{FAI}_{2011}=$ $+0,286$ e $\mathrm{FAI}_{2012}=0,000$.

Devido à baixa variação observada entre $\mathrm{CMS}_{\mathrm{OBS}}$ e $\mathrm{CMS}_{\mathrm{EST}}$ nos dois anos de avaliação, optou-se por dividir os animais em três classes de eficiência, cujos limites são $\pm 75 \%$ do desvio padrão (DP) registrado para o CAR. Dessa forma, os animais foram classificados como eficientes $\left(\mathrm{CAR}<-0,75 * \mathrm{DP}_{\mathrm{CAR}}\right)$, intermediários $\left(-0,75 * \mathrm{DP}_{\mathrm{CAR}}<\mathrm{CAR}<+0,75 * \mathrm{DP}_{\mathrm{CAR}}\right) \mathrm{e}$ ineficientes $\left(\mathrm{CAR}>+0,75^{*} \mathrm{DP}_{\mathrm{CAR}}\right)$.

Os dados foram submetidos à análise de variância pelo modelo linear geral (PROC GLM) em delineamento inteiramente casualizado com três tratamentos, que corresponderam às classes de CAR. As médias que apresentaram diferença significativa $(\mathrm{P}<0,05)$ entre as classes foram comparadas pelo teste de Tukey-Kramer. Análises de correlação simples de Pearson (PROC CORR) foram realizadas com CAR e as demais características de desempenho, consumo e eficiência alimentar. As análises estatísticas foram realizadas utilizando-se o programa SAS. 


\section{Fernandes et al.}

\section{RESULTADOS E DISCUSSÃO}

O número de animais em cada classe de eficiência correspondeu a 25, 59 e 26 nas classes eficiente (CAR baixo), intermediária (CAR médio) e ineficiente (CAR alto), respectivamente (Tab. 2). Essa desigualdade no número de animais em cada classe está relacionada ao critério de classificação utilizado neste estudo, que foi $\pm 75 \%$ do DP registrado para o CAR. Na maioria dos estudos em que o CAR é utilizado como critério de classificação de bovinos eficientes, intermediários e ineficientes, o limite das classes é $\pm 50 \%$ do DP do CAR (Basarab et al., 2003; Lucila Sobrinho et al., 2011; Santana et al., 2012). Nessa condição, o número de animais distribuídos em cada classe é uniforme, com frequência relativa próxima de $33 \%$ dos animais avaliados em cada classe.

Tabela 2. Médias e erro padrão das características de desempenho, consumo e eficiência alimentar de touros Purunã em crescimento nas três classes de eficiência determinadas com base no consumo alimentar residual (CAR)

\begin{tabular}{|c|c|c|c|c|}
\hline \multirow[b]{2}{*}{ Característica $^{1}$} & \multicolumn{3}{|c|}{ Classes de Eficiência } & \multirow[b]{2}{*}{$\mathrm{P}$} \\
\hline & $\begin{array}{c}\text { Eficiente } \\
\text { (CAR baixo) }\end{array}$ & $\begin{array}{l}\text { Intermediária } \\
\text { (CAR médio) }\end{array}$ & $\begin{array}{l}\text { Ineficiente } \\
\text { (CAR alto) }\end{array}$ & \\
\hline Número de animais (n) & 25 & 59 & 26 & - \\
\hline Peso corporal inicial (kg) & $238,1 \pm 8,5$ & $230,6 \pm 5,6$ & $233,7 \pm 8,4$ & 0,7605 \\
\hline Peso corporal final (kg) & $363,4 \pm 10,5$ & $358,9 \pm 6,8$ & $359,7 \pm 10,3$ & 0,9362 \\
\hline PMM $\left(\mathrm{kg}^{0,75}\right)$ & $72,0 \pm 1,7$ & $71,0 \pm 1,1$ & $71,3 \pm 1,6$ & 0,8732 \\
\hline GMD (kg/dia) & $1,12 \pm 0,04$ & $1,15 \pm 0,03$ & $1,12 \pm 0,04$ & 0,8415 \\
\hline $\mathrm{CMS}_{\mathrm{OBS}}(\mathrm{kg} / \mathrm{dia})$ & $7,19 \pm 0,20 b$ & $7,62 \pm 0,13 \mathrm{ab}$ & $8,10 \pm 0,19 a$ & 0,0066 \\
\hline $\mathrm{CMS}_{\mathrm{PC}}(\% \mathrm{PC} / \mathrm{dia})$ & $2,41 \pm 0,04 \mathrm{c}$ & $2,60 \pm 0,03 \mathrm{~b}$ & $2,74 \pm 0,04 \mathrm{a}$ & 0,0000 \\
\hline $\mathrm{CMS}_{\mathrm{PMM}}\left(\% \mathrm{~kg}^{0,75} / \mathrm{dia}\right)$ & $9,98 \pm 0,14 \mathrm{c}$ & $10,74 \pm 0,09 b$ & $11,35 \pm 0,14 \mathrm{a}$ & 0,0000 \\
\hline $\mathrm{CMS}_{\text {MANT }}(\mathrm{kg} / \mathrm{dia})$ & $3,34 \pm 0,06 b$ & $3,46 \pm 0,04 b$ & $3,67 \pm 0,06 \mathrm{a}$ & 0,0010 \\
\hline $\mathrm{CMS}_{\mathrm{GANHO}}(\mathrm{kg} / \mathrm{dia})$ & $3,86 \pm 0,17$ & $4,16 \pm 0,11$ & $4,42 \pm 0,17$ & 0,0631 \\
\hline $\mathrm{CMS}_{\mathrm{EST}}(\mathrm{kg} / \mathrm{dia})$ & $7,68 \pm 0,19$ & $7,63 \pm 0,13$ & $7,62 \pm 0,19$ & 0,9718 \\
\hline CAR (kg MS/dia) & $-0,48 \pm 0,04 c$ & $-0,01 \pm 0,02 \mathrm{~b}$ & $0,48 \pm 0,04 \mathrm{a}$ & 0,0000 \\
\hline CA (kg MS/kg ganho) & $6,50 \pm 0,21 b$ & $6,80 \pm 0,14 b$ & $7,40 \pm 0,21 \mathrm{a}$ & 0,0096 \\
\hline EAB (g ganho/kg MS) & $156,2 \pm 3,9 \mathrm{a}$ & $150,1 \pm 2,6 \mathrm{a}$ & $137,9 \pm 3,9 b$ & 0,0040 \\
\hline EMant (g ganho/kg MS para mantença) & $338,0 \pm 14,1$ & $334,8 \pm 9,2$ & $308,3 \pm 13,9$ & 0,2240 \\
\hline EPC (g ganho/kg MS para crescimento) & $294,1 \pm 4,8 \mathrm{a}$ & $277,0 \pm 3,1 \mathrm{~b}$ & $254,5 \pm 4,7 \mathrm{c}$ & 0,0000 \\
\hline Taxa de Crescimento Relativo (\%/dia) & $0,17 \pm 0,01$ & $0,17 \pm 0,00$ & $0,17 \pm 0,01$ & 0,6269 \\
\hline Taxa de Kleiber $\left(\mathrm{kg}\right.$ ganho $\left./ \mathrm{kg}^{0,75}\right)$ & $0,016 \pm 0,001$ & $0,016 \pm 0,000$ & $0,016 \pm 0,001$ & 0,5907 \\
\hline
\end{tabular}

${ }^{1} \mathrm{PMM}$ : peso médio metabólico; GMD: ganho médio diário; $\mathrm{CMS}_{\mathrm{PC}}$ : consumo de matéria seca (MS) em relação ao peso corporal (PC); $\mathrm{CMS}_{\mathrm{PMM}}$ : consumo de MS em relação ao PMM; $\mathrm{CMS}_{\mathrm{MANT}}$ : consumo de MS estimado para mantença; $\mathrm{CMS}_{\mathrm{GANHO}}$ : consumo de MS estimado para crescimento; $\mathrm{CMS}_{\mathrm{OBS}}$ : consumo de MS observado; $\mathrm{CMS}_{\mathrm{EST}}$ : consumo de MS estimado com base no GMD e PMM; CAR: consumo alimentar residual; CA: conversão alimentar; EAB: eficiência alimentar bruta; EMant: eficiência de mantença; EPC: eficiência parcial de crescimento.

Médias seguidas por letras minúsculas na mesma linha diferem pelo teste Tukey-Kramer $(\mathrm{P}<0,05)$.

Dada a baixa variação entre $\mathrm{CMS}_{\mathrm{OBS}}$ e $\mathrm{CMS}_{\mathrm{EST}}$ na população de tourinhos Purunã avaliada, optou-se por um critério de classificação mais rigoroso, com o objetivo de identificar e isolar os animais eficientes e ineficientes para CAR em classes mais restritas, porém com características de eficiência alimentar bastante distintas. Por esse critério, registrou-se frequência relativa de 22,7; 53,6 e 23,6\% dos tourinhos avaliados nas classes eficiente, intermediária e ineficiente, respectivamente.
O PC inicial e final, o PMM e o GMD foram semelhantes entre as três classes de eficiência (Tab. 2). Os valores médios dessas características corresponderam a 233 e $360 \mathrm{~kg}$ para PC inicial e final, $71,3 \mathrm{~kg}$ para PMM e 1,14kg/dia para GMD. A semelhança dessas características entre as três classes era esperada, pois a classificação de animais com menor e maior eficiência alimentar com base no CAR não altera o PC, o PMM e o GMD (Herd e Arthur, 2009). Isso é confirmado pelos valores nulos e não significativos obtidos 
para correlação entre CAR e PC inicial e final, PMM e GMD (Tab. 3).

O $\mathrm{CMS}_{\mathrm{OBS}}$ foi menor na classe eficiente $(7,19 \mathrm{~kg} / \mathrm{dia})$ e maior na classe ineficiente $(8,10 \mathrm{~kg} / \mathrm{dia})$, enquanto a classe intermediária foi semelhante às demais $(7,62 \mathrm{~kg} / \mathrm{dia} ;$ Tab. 2). O $\mathrm{CMS}_{\mathrm{EST}}$ não diferiu entre as classes de eficiência (Tab. 2) e apresentou média de 7,64kg/dia. A equação obtida pela relação entre $\mathrm{CMS}_{\mathrm{OBS}} \mathrm{e}$ $\mathrm{CMS}_{\mathrm{EST}}$ apresentou $\mathrm{R}^{2}=0,87$ (Fig. 1), e indicou que as variáveis inclusas no modelo de predição de consumo de MS explicam $87 \%$ da variação observada entre $\mathrm{CMS}_{\mathrm{OBS}}$ e $\mathrm{CMS}_{\mathrm{EST}}$ para os tourinhos avaliados. O PMM, o GMD e o FAI $_{\text {ANO }}$ explicam 67,7; 18,1 e 1,2\% dessa variação, enquanto os $13 \%$ restantes são explicados por outros fatores biológicos.
A variação entre $\mathrm{CMS}_{\mathrm{OBS}}$ e o $\mathrm{CMS}_{\mathrm{EST}}$ registrada para cada animal corresponde à distância vertical de cada ponto de $\mathrm{CMS}_{\mathrm{OBS}}$ até a linha de regressão (Fig. 1), que, por definição, é o CAR. A média e o DP dessa característica foi $0,00 \pm$ $0,37 \mathrm{~kg} \mathrm{MS} /$ dia. Essa variação foi inferior à relatada por Basarab et al. (2003) e Nkrumah et al. (2004), que trabalharam com novilhos cruzados europeus, e por Lancaster et al. (2009), que avaliaram novilhos puros da raça Angus. Os valores de DP observados nesses estudos foram 0,$66 ; 0,83$ e $0,78 \mathrm{~kg} \mathrm{MS} / \mathrm{dia}$, respectivamente. A baixa variação do CAR no presente estudo pode estar relacionada à baixa variabilidade genética dos animais utilizados, uma vez que todos os tourinhos Purunã eram provenientes do local em que a raça foi desenvolvida.

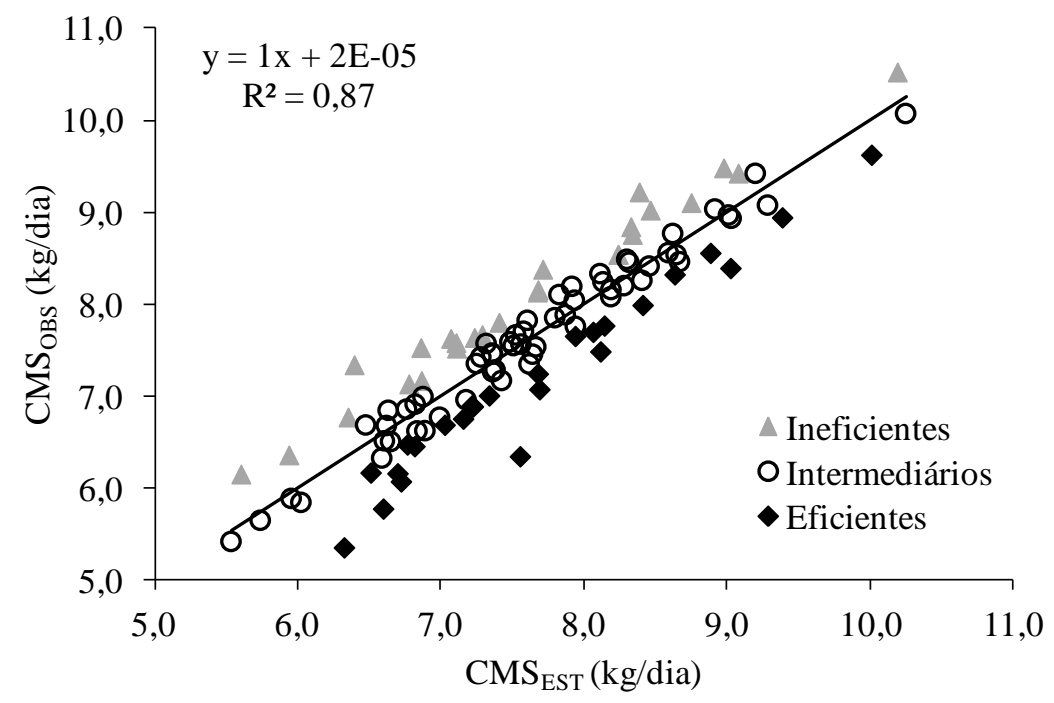

Figura 1. Relação entre consumo de matéria seca observado $\left(\mathrm{CMS}_{\mathrm{OBS}}\right)$ e estimado $\left(\mathrm{CMS}_{\mathrm{EST}}\right)$ para touros Purunã em crescimento de três classes de eficiência determinadas com base no consumo alimentar residual (CAR).

O CAR apresentou valor mínimo de $-1,21 \mathrm{~kg}$ MS/dia e máximo de $0,95 \mathrm{~kg}$ MS/dia, o que representa uma diferença de $2,16 \mathrm{~kg}$ MS/dia entre o animal mais e o menos eficiente. Basarab et al. (2003), Nkrumah et al. (2004) e Lancaster et al. (2009) observaram maiores amplitudes de variação para CAR, com valores de 3,77; 4,86 e $5,04 \mathrm{~kg} \mathrm{MS} /$ dia, respectivamente. A capacidade de digestão do alimento e de absorção dos nutrientes, o incremento calórico associado à alimentação, a composição corporal e os processos metabólicos que ocorrem em nível tecidual (visceral, muscular e adiposo), e a intensidade de atividade física são os fatores que exercem maior influência no consumo de alimento e, por consequência, no CAR (Herd e Arthur, 2009).

A composição corporal pode ser considerada um potencial fator de influência do CAR em animais da raça Purunã, pois é formada por cruzamentos entre raças que apresentam diferentes características de crescimento, principalmente na composição do ganho de peso. Entre estas, destacam-se as raças Charolês e Canchim, com alto potencial de ganho de peso e 
desenvolvimento muscular (Kuss et al., 2008), e a raça Angus, com precocidade na deposição de gordura na carcaça (Goulart et al., 2008). Provavelmente, tourinhos que consumiram maior quantidade de alimento e foram classificados como ineficientes apresentaram maior deposição de gordura na carcaça, que é positivamente correlacionada com o CAR (Nkrumah et al., 2004; Lancaster et al., 2009).

O valor médio de CAR nas classes eficiente, intermediária e ineficiente foi $-0,48 ;-0,01$ e $0,48 \mathrm{~kg} \mathrm{MS} /$ dia, respectivamente (Tab. 2). A diferença entre o valor médio de CAR das classes eficiente e ineficiente indicou que tourinhos eficientes consumiram, em média, $0,96 \mathrm{~kg} \mathrm{MS} / \mathrm{dia}$ a menos que os ineficientes. Considerando o teor médio de MS do concentrado $(884,6 \mathrm{~g} / \mathrm{kg} \mathrm{MN})$ e da silagem de milho $(283,9 \mathrm{~g} / \mathrm{kg} \mathrm{MN})$ ofertados nos dois anos de avaliação (Tab. 1), e a relação volumoso:concentrado da dieta $(58: 42)$, a diferença no consumo de MS entre tourinhos eficientes e ineficientes correspondeu, em base de $\mathrm{MN}$, a $0,46 \mathrm{~kg} / \mathrm{dia}$ de concentrado e $1,96 \mathrm{~kg} / \mathrm{dia}$ de silagem de milho, totalizando $2,42 \mathrm{~kg} / \mathrm{dia}$ da dieta total. Assim, o consumo de alimento por animal na classe eficiente foi $271 \mathrm{~kg}$ inferior à classe ineficiente no período experimental, o que evidencia o impacto positivo dos animais de CAR negativo na redução dos custos com alimentação.

A semelhança do PC e GMD entre as classes de eficiência, e o menor consumo de alimento observado na classe eficiente (Tab. 2) mostram que animais eficientes para CAR podem melhorar os índices zootécnicos e econômicos nos sistemas de produção em que estão inseridos. As estimativas de herdabilidade para CAR em bovinos de raças taurinas em fase de crescimento são moderadas e variam de 0,16 a 0,43 (Herd et al., 2003). Portanto, a variação genética para CAR em bovinos da raça Purunã deve ocorrer e pode ser considerada na seleção de touros para reprodução, buscando melhorar a eficiência alimentar sem afetar as características produtivas da progênie.

O $\mathrm{CMS}_{\mathrm{PC}}$ e o $\mathrm{CMS}_{\mathrm{PMM}}$ apresentaram valores médios de 2,59\% PC/dia e 10,71\% PMM/dia e diferiram entre as classes de eficiência (Tab. 2). Como o PC médio dos animais foi semelhante entre as classes, as medidas de consumo de
MS expressas em relação ao PC e PMM acompanharam o $\mathrm{CMS}_{\mathrm{OBS}}$. Os valores de $\mathrm{CMS}_{\mathrm{PC}}$ foram 2,$41 ; 2,60$ e $2,74 \%$ PC/dia, e de $\mathrm{CMS}_{\mathrm{PMM}}$ foram 9,98; 10,74 e $11,35 \%$ PMM/dia para tourinhos eficientes, intermediários e ineficientes, respectivamente.

Registraram-se valores médios de 3,48 e $4,15 \mathrm{~kg} /$ dia para $\mathrm{CMS}_{\mathrm{MANT}}$ e $\mathrm{CMS}_{\mathrm{GANHO}}$, sendo que apenas o primeiro diferiu entre as classes de eficiência (Tab. 2). O $\mathrm{CMS}_{\mathrm{MANT}}$ foi maior na classe ineficiente $(3,67 \mathrm{~kg} / \mathrm{dia})$ e menor nas classes intermediária e eficiente, que foram semelhantes entre si (média de $3,40 \mathrm{~kg} / \mathrm{dia}$ ). Apesar de não ter diferido estatisticamente $(\mathrm{P}=$ 0,0631), o $\mathrm{CMS}_{\mathrm{GANHO}}$ na classe ineficiente $(4,42 \mathrm{~kg} / \mathrm{dia})$ foi numericamente superior em $0,56 \mathrm{~kg} /$ dia à classe eficiente $(3,86 \mathrm{~kg} / \mathrm{dia})$.

O consumo de MS para atender às necessidades de mantença e crescimento em tourinhos ineficientes foram superiores em 0,33 e $0,56 \mathrm{~kg}$ MS/dia aos tourinhos eficientes. Essas diferenças podem estar relacionadas a processos metabólicos, entre eles a taxa de turnover proteico. Animais com baixas taxas de turnover proteico no tecido muscular têm menores necessidades de ingestão de energia para mantença, o que parece ocorrer em animais eficientes para CAR (Castro Bulle et al., 2007). Provavelmente, tourinhos Purunã eficientes despenderam menos energia em processos metabólicos, o que levou a menor necessidade de consumo de MS para mantença e crescimento.

A CA e a EAB diferiram entre as classes de eficiência e foram influenciadas pelo $\mathrm{CMS}_{\mathrm{OBS}}$ (Tab. 2). A CA foi semelhante entre as classes eficiente e intermediária (média de $6,65 \mathrm{~kg}$ $\mathrm{MS} / \mathrm{kg}$ ganho), que foram inferiores à classe ineficiente $(7,40 \mathrm{~kg} \mathrm{MS} / \mathrm{kg}$ ganho). Já a EAB apresentou resultado inverso ao da $\mathrm{CA}$, com valores inferiores na classe ineficiente (138 g ganho/kg MS) e superiores nas classes eficiente e intermediária, que não diferiram entre si e apresentaram média de $153 \mathrm{~g}$ ganho/kg MS. Dessa forma, tourinhos das classes eficiente e intermediária consumiram $0,75 \mathrm{~kg} \mathrm{MS} / \mathrm{kg}$ ganho a menos que os da classe ineficiente, o que correspondeu ao aumento de $15 \mathrm{~g}$ ganho/ $\mathrm{kg}$ MS na $\mathrm{EAB}$ dos primeiros.

A EMant foi semelhante entre as classes de eficiência (Tab. 2) e apresentou média de $329 \mathrm{~g}$ 
ganho/kg MS para mantença. Já a EPC diferiu entre as classes, com valores médios de 294; 277 e $255 \mathrm{~g}$ ganho/kg MS para crescimento nas classes eficiente, intermediária e ineficiente (Tab. 2). Essas medidas de eficiência estabelecem a relação entre GMD e o consumo de MS para atender as necessidades de mantença $\left(\mathrm{CMS}_{\mathrm{MANT}}\right)$ e crescimento $\left(\mathrm{CMS}_{\mathrm{GANHO}}\right)$. Apesar de o $\mathrm{CMS}_{\mathrm{MANT}}$ ter sido maior na classe ineficiente, a relação entre GMD e essa variável de consumo foi equivalente nas três classes de eficiência, determinando valores semelhantes de EMant entre as mesmas. Por outro lado, a EPC mostrou-se inversamente proporcional ao aumento de $\mathrm{CMS}_{\mathrm{GANHO}}$ da classe eficiente para a ineficiente. Assim, tourinhos eficientes têm EMant semelhante e EPC superior em 17 e $40 \mathrm{~g}$ ganho/kg MS para crescimento aos tourinhos intermediários e ineficientes para CAR, respectivamente.

As taxas de crescimento relativo e de Kleiber foram semelhantes entre as classes de eficiência (Tab. 2) e apresentaram valores médios de 0,17 e 0,016, respectivamente. Essas medidas de eficiência são dependentes do PC inicial e final, do PMM e do GMD. Com exceção do PC final, essas características apresentaram correlação significativa com as taxas de crescimento relativo ( $\mathrm{PC}$ inicial, $\mathrm{r}=-0,61 ; \mathrm{PMM}, \mathrm{r}=-0,38$; GMD, $r=0,69$ ) e de Kleiber (PC inicial, $r=-$ 0,44; PMM, r = -0,19; GMD, r = 0,81) (Tab. 3). Como o PC e o GMD não diferiram entre as classes, as taxas de crescimento relativo e de Kleiber apresentaram valores equivalentes entre as mesmas.

Tabela 3. Correlações simples de Pearson entre as características de desempenho, consumo e eficiência alimentar de touros Purunã em crescimento

\begin{tabular}{|c|c|c|c|c|c|c|c|c|c|c|c|}
\hline Característica $^{1}$ & PF & PMM & GMD & $\mathrm{CMS}_{\mathrm{OBS}}$ & CAR & CA & EAB & EMant & $\mathrm{EPC}$ & TCR & TK \\
\hline PI & $0,88 * * *$ & $0,96 * * *$ & $0,14^{\mathrm{NS}}$ & $0,68 * * *$ & $0,00^{\mathrm{NS}}$ & $0,42 * * *$ & $-0,46 * * *$ & $-0,25 * *$ & $-0,72 * * *$ & $-0,61 * * *$ & $-0,44 * * *$ \\
\hline $\mathrm{PF}$ & & $0,98 * * *$ & $0,59^{* * *}$ & $0,89^{* * *}$ & $0,00^{\mathrm{NS}}$ & $0,00^{\mathrm{NS}}$ & $-0,04^{\mathrm{NS}}$ & $0,22 *$ & $-0,55^{* * *}$ & $-0,17^{\mathrm{NS}}$ & $0,02^{\mathrm{NS}}$ \\
\hline PMM & & & $0,40^{* * *}$ & $0,82 * * *$ & $0,00^{\mathrm{NS}}$ & $0,20^{*}$ & $-0,24 *$ & $0,01^{\mathrm{NS}}$ & $-0,65 * * *$ & $-0,38 * * *$ & $-0,19^{*}$ \\
\hline GMD & & & & $0,72 * * *$ & $0,00^{\mathrm{NS}}$ & $-0,72 * * *$ & $0,72 * * *$ & $0,90^{* * *}$ & $0,09^{\mathrm{NS}}$ & $0,69^{* * *}$ & $0,81^{* * *}$ \\
\hline $\mathrm{CMS}_{\mathrm{OBS}}$ & & & & & $0,36 * *$ & $-0,07^{\mathrm{NS}}$ & $0,04^{\mathrm{NS}}$ & $0,35^{* *}$ & $-0,62 * * *$ & $0,08^{\mathrm{NS}}$ & $0,26^{* *}$ \\
\hline CAR & & & & & & $0,35 * *$ & $-0,37 * * *$ & $-0,18^{\mathrm{NS}}$ & $-0,62 * * *$ & $0,00^{\mathrm{NS}}$ & $0,02^{\mathrm{NS}}$ \\
\hline $\mathrm{CA}$ & & & & & & & $-0,98^{* * * *}$ & $-0,91$ **** & $-0,71 * * *$ & $-0,88 * * *$ & $-0,88^{* * *}$ \\
\hline EAB & & & & & & & & $0,94 * * *$ & $0,74^{* * *}$ & $0,91^{* * *}$ & $0,91^{* * *}$ \\
\hline EMant & & & & & & & & & $0,48 * * *$ & $0,90 * * *$ & $0,95^{* * *}$ \\
\hline $\mathrm{EPC}$ & & & & & & & & & & $0,59^{* * *}$ & $0,48^{* * * *}$ \\
\hline TCR & & & & & & & & & & & $0,98^{* * *}$ \\
\hline
\end{tabular}

${ }^{1}$ PI: peso corporal inicial; PF: peso corporal final; PMM: peso médio metabólico; GMD: ganho médio diário; $\mathrm{CMS}_{\mathrm{OBS}}$ : consumo de matéria seca observado; CAR: consumo alimentar residual; CA: conversão alimentar; EAB: eficiência alimentar bruta; EMant: eficiência de mantença; EPC: eficiência parcial de crescimento; TCR: taxa de crescimento relativo; TK: taxa de Kleiber.

Significância: NS: não significativo, $\mathrm{P}>0,05 ; * \mathrm{P}<0,05 ; * * \mathrm{P}<0,01 ; * * * \mathrm{P}<0,0001$.

A análise de correlação entre as medidas de eficiência alimentar (Tab. 3) mostrou que houve correlação significativa entre CAR e CA $(\mathrm{r}=$ $0,35)$, $\operatorname{EAB}(r=-0,37)$ e $\operatorname{EPC}(r=-0,62)$. As correlações entre essas características foram moderadas (CA e EAB) a altas (EPC), e indicam que a diminuição do $\mathrm{CAR}$ leva à redução da $\mathrm{CA}$ e ao aumento da EAB e da EPC. Ao relacionar os valores médios de CA, EAB e EPC da classe eficiente com os da classe ineficiente (Tab. 2), observou-se que a diminuição do CAR ocasionou redução da CA em $12,2 \%$, e aumento da $\mathrm{EAB}$ e da EPC em 13,3 e 15,5\%, respectivamente. A melhoria dessas medidas de eficiência em relação ao CAR é explicada pela inalteração do
PC e do GMD associada à diminuição do consumo de MS à medida que o CAR diminui. Além disso, a alta correlação entre CAR e EPC indica que a diminuição do CAR está relacionada à menor necessidade de consumo de MS para crescimento. Estudos realizados com bovinos de raças taurinas em fase de crescimento mostram que a correlação entre CAR e EPC pode variar de -0,65 a -0,89 (Arthur et al., 2001; Nkrumah et al., 2004; Lancaster et al., 2009).

As demais medidas de eficiência alimentar foram correlacionadas entre si, com valores de correlação moderados a altos (Tab. 3). No entanto, todas essas medidas têm correlação 
significativa com peso corporal (PI e PF) e/ou ganho de peso (GMD), o que indica que mudanças na eficiência alimentar com base em CA, EAB, EMant, EPC, taxa de crescimento relativo e taxa de Kleiber alteram o PC e o GMD dos animais.

\section{CONCLUSÕES}

Tourinhos Purunã classificados como eficientes para consumo alimentar residual apresentam menor consumo de alimento, mas mantêm o mesmo nível de produção dos classificados como ineficientes. Melhor conversão alimentar, maior eficiência alimentar bruta e maior eficiência parcial de crescimento são observados em tourinhos eficientes para consumo alimentar residual. Assim, a classificação com base nessa característica de eficiência alimentar permite a identificação de animais com potencial para melhorar a eficiência produtiva do rebanho.

\section{REFERÊNCIAS}

ARTHUR, P.F.; RENAND, G.; KRAUSS, D. Genetic and phenotypic relationships among different measures of growth and feed efficiency in young Charolais bulls. Livest. Prod. Sci., v.68, p.131-139, 2001.

BASARAB, J.A.; PRICE, M.A.; AALHUS, J.L. et al. Residual feed intake and body composition in young growing cattle. Can. J. Anim. Sci., v.83, p.189-204, 2003.

CASTRO BULLE, F.C.P.; PAULINO, P.V.; SANCHES, A.C.; SAINZ, R.D. Growth, carcass quality, and protein and energy metabolism in beef cattle with different growth potentials and residual feed intakes. J. Anim. Sci., v.85, p.928-936, 2007.

FITZHUGH JUNIOR, H.A.; TAYLOR, St.C.S. Genetic analysis of degree of maturity. J. Anim. Sci., v.33, p.717-725, 1971

GOULART, R.S.; ALENCAR, M.M.; POTT, E.B. et al. Composição corporal e exigências líquidas de proteína e energia de bovinos de quatro grupos genéticos terminados em confinamento. Rev. Bras. Zootec., v.37, p.926-935, 2008.

HEGARTY, R.S.; GOOPY, J.P.; HERD, R.M. et al. Cattle selected for lower residual feed intake have reduced daily methane production. J. Anim. Sci., v.85, p.1479-1486, 2007.

HERD, R.M.; ARCHER, J.A.; ARTHUR, P.F. Reducing the cost of beef production through genetic improvement in residual feed intake: Opportunity and challenges to application. J. Anim. Sci., v.81, p.E9E17, 2003.
HERD, R.M.; ARTHUR, P.F. Physiological basis for residual feed intake. J. Anim. Sci., v.87, p.E64-E71, 2009.

KLEIBER, M. Body size and metabolic rate. Physiol. Rev., v.27, p.511-541, 1947.

KOCH, R.M.; SWIGER, L.A.; CHAMBERS, D.; GREGORY, K.E. Efficiency of feed use in beef cattle. J. Anim. Sci., v.22, p.486-494, 1963.

KUSS, F.; MOLETTA, J.L.; PEROTTO, D. et al. Carcaça e carne de novilhos cruzas Pardo Suíço x Canchim e Purunã $x$ Canchim terminados em confinamento. Cienc. Rural, v.38, p.1061-1066, 2008.

LANCASTER, P.A.; CARSTENS, G.E.; RIBEIRO, F.R.B. et al. Characterization of feed efficiency traits and relationships with feeding behavior and ultrasound carcass traits in growing bulls. J. Anim. Sci., v.87, p.1528-1539, 2009 .

LOFGREEN, G.P.; GARRETT, W.N. A system for expressing net energy requirements and feed values for growing and finishing beef cattle. J. Anim. Sci., v. 27, p.793-806, 1968.

LUCILA SOBRINHO, T.; BRANCO, R.H.; BONILHA, S.F.M. et al. Residual feed intake and relationships with performance of Nellore cattle selected for post weaning weight. Rev. Bras. Zootec., v.40, p.929-937, 2011.

NKRUMAH, J.D.; BASARAB, J.A.; PRICE, M.A. et al. Different measures of energetic efficiency and their phenotypic relationships with growth, feed intake, and ultrasound and carcass merit in hybrid cattle. J. Anim. Sci., v.82, p.2451-2459, 2004.

NATIONAL RESEARCH COUNCIL - NRC. Nutrient requirements of dairy cattle. 7.rev.ed. Washington: National Academy Press, 2001. 381p.

SILVA, D.J.; QUEIROZ, A.C. Análise de alimentos: métodos químicos e biológicos. Viçosa, MG: Universidade Federal de Viçosa, 2002. 235p.

SANTANA, M.H.A.; ROSSI JUNIOR, P.; ALMEIDA, R.; CUCCO, D.C. Feed efficiency and its correlations with carcass traits measured by ultrasound in Nellore bulls. Livest. Sci., v.145, p.252-257, 2012.

VAN SOEST, P.J.; ROBERTSON, J.B.; LEWIS, B.A. Methods for dietary fiber, neutral detergent fiber, and no starch polysaccharides in relation to animal nutrition. J. Dairy Sci., v.74, p.3583-3597, 1991.

ZINN, A.; SHEN, Y.A. An evaluation of ruminal degradable intake protein and metabolizable amino acid requirements of feedlot calves. J. Anim. Sci., v.76, p.1280-1289, 1998. 